\title{
Precipitation of dichloromethane as low-chlorine carbon nanoparticles from water by femtosecond laser pulses
}

\section{Takuya Okamoto, Erina Miyasaka, Koji Mitamura, Kimihiro Matsukawa and Tomoyuki Yatsuhashi}

\begin{tabular}{|c|l|}
\hline Citation & Journal of Photochemistry \& Photobiology, A: Chemistry, 344; 178-183 \\
\hline Issue Date & $2017-07-01$ \\
\hline Type & Journal Article \\
\hline Textversion & Author \\
\hline Highlights & $\begin{array}{l}\text {-Volatile organochlorine compounds in water are precipitated as carbon } \\
\text { nanoparticles. }\end{array}$ \\
\hline Remote treatment of organic wastes is achieved by femtosecond laser irradiations. \\
Rights & $\begin{array}{l}\text { C } 2017 \text { Elsevier B.V. This work is licensed under a Creative Commons } \\
\text { Attribution-NonCommercial-NoDerivatives } 4.0 \text { International License. } \\
\text { http://creativecommons.org/licenses/by-nc-nd/4.0/ }\end{array}$ \\
& $\begin{array}{l}\text { The article has been published in final form at } \\
\text { https://doi.org/10.1016/j.jphotochem.2017.05.011 }\end{array}$ \\
\hline DOI & \begin{tabular}{l}
$10.1016 / j . j p h o t o c h e m .2017 .05 .011$ \\
\hline
\end{tabular} \\
\hline
\end{tabular}

Self-Archiving by Author(s)

Placed on: Osaka City University

OKAMOTO, T., MIYASAKA, E., MITAMURA, K., MATSUKAWA, K., \& YATSUHASHI, T. (2017). Precipitation of dichloromethane as low-chlorine carbon nanoparticles from water by femtosecond laser pulses. Journal of Photochemistry \& Photobiology, A: Chemistry. 344, 178-183. 


\section{Precipitation of dichloromethane as low-chlorine}

\section{carbon nanoparticles from water by femtosecond}

\section{laser pulses}

Takuya Okamoto, ${ }^{\dagger}$ Erina Miyasaka, ${ }^{\dagger}$ Koji Mitamura, ${ }^{,}$Kimihiro Matsukawa, ${ }^{\ddagger}$ Tomoyuki

Yatsuhashi*广

Department of Chemistry, Graduate School of Science, Osaka City University, 3-3-138

Sugimoto, Sumiyoshi, Osaka 558-8585, Japan, Osaka Municipal Technical Research Institute, 1-6-50 Morinomiya, Joto, Osaka 536-8553, Japan.

* To whom correspondence should be addressed. Telephone: +81-6-6605-2554. FAX: +81-66605-2522. E-mail: tomo@sci.osaka-cu.ac.jp (T.Y.)

${ }^{\dagger}$ Graduate School of Science, Osaka City University.

Osaka Municipal Technical Research Institute 


\begin{abstract}
We report on the transformation of dichloromethane to low-chlorine carbon nanoparticles by the exposure of aqueous dichloromethane solution and/or dichloromethane/water biphasic solution to femtosecond laser pulses. The $\mathrm{pH}$ of either solution immediately decreased due to the reduction of dichloromethane by hydrated electrons. The time evolution of transmittance revealed that particle formation was suppressed when the initial $\mathrm{pH}$ was low because protons scavenge hydrated electrons. The size and morphology of nanoparticles was independent of the initial $\mathrm{pH}$ and the reaction solution used. The analysis of the elemental composition of carbon particles showed that the $\mathrm{Cl} / \mathrm{C}$ atomic ratio was, independent on the initial $\mathrm{pH}$ values, greatly reduced to 0.06 . We propose that the violent destruction of dichloromethane and elimination of chlorine atoms not only as $\mathrm{Cl}^{-}$but also $\mathrm{Cl}_{2}$ excludes chlorine atoms from the carbon network construction processes toward the formation of carbon nanoparticles.
\end{abstract}

\title{
Keywords
}

ionization; hydrated electrons; filamentation, dechlorination, remote treatment 


\section{Introduction}

Chlorohydrocarbons (CHCs), which are widely used in the chemical industry, are regarded as environmental pollutants due to their highly volatile nature and high solubility in water [1]. Emission of CHCs into the atmosphere and their discharge into water are known to harm the global environment. For the treatment of massive CHC fluid, combustion has practically been used. To remove CHCs from water, physical methods such as adsorption and membrane filtering have been used, as have biological [1] and catalytic treatments [2]. In addition, sonication [3], vacuum ultraviolet light irradiation [4], and radiolysis [5] have been examined to decompose CHCs in water. However, difficulties arise in those methods if the complete mineralization is not achieved. Once more volatile and soluble chlorine-containing organic fragments are formed from CHCs, it is difficult to separate them from water. For example, products originating in radical recombination, such as di-, tri-, and tetrachloroethane are found by VUV photolysis of aqueous dichloromethane (DCM) solution [4]. $\mathrm{C}_{2} \mathrm{H}_{4} \mathrm{Cl}_{2}, \mathrm{HCOOH}$, and $\mathrm{CH}_{2} \mathrm{O}$ are obtained by $\gamma$-radiolysis of DCM in water [6]. The treatment of CHCs dissolved in water by above mentioned methods is based on the production of reactive species such as hydrated electrons $\left(\mathrm{e}^{-}\right.$aq) and hydroxyl radicals $(\bullet \mathrm{OH})$ produced by high-energy deposition into water [2-6]. However, it seems that the conventional methods cannot produce enough density of reactive species to achieve complete decomposition of DCM. Herein, we suggest that the alternative way to remove CHCs from water is precipitation of the products of $\mathrm{CHCs}$ in water. If we obtain the resultant products as solid forms, the filtering of those products from water can be done easily. The formation of precipitates from aqueous DCM solution has only been reported in the case of high-dose radiolysis [5]. However, the resultant polymer contains a large amount of chlorine $(\mathrm{Cl} / \mathrm{C}=0.34$, stoichiometry was $\mathrm{C}_{3} \mathrm{H}_{3} \mathrm{OCl}$ [5].) The establishment of the simultaneous achievements of both 
dechlorination and solidification is expected to meet the requirement of the alternative methods for the treatment of CHCs.

It is well known that focusing intense femtosecond laser pulses onto water creates a high density of $\mathrm{e}^{-}$aq and $\bullet \mathrm{OH}$ in a well-confined volume [7]. Recently, we synthesized hydrophilic and hydrophobic carbon nanoparticles (CNPs) by femtosecond laser irradiation of the water phase of an aerated benzene/water biphasic solution as well as of the aqueous benzene solution $[7,8]$. We suggested that successive oxidations of benzene with $\bullet \mathrm{OH}$ finally lead to the formation of CNPs and their agglomerates [8]. In the case of benzene, the reaction rate of benzene with $\mathrm{e}^{-}$aq is much smaller than that with $\bullet \mathrm{OH}$. On the contrary, we have shown that metal ions were reduced by a high density of $\mathrm{e}^{-}$aq generated from solvent by femtosecond laser ionization [9-12].

In this study, we report the significant reduction of $\mathrm{Cl} / \mathrm{C}$ ratio from 2 (the reactant, $\mathrm{DCM}$ ) to 0.06 (the products) are achieved by the transformation of DCM to carbon nanoparticles (CNPs) by femtosecond laser pulse irradiations. The relatively small $\mathrm{Cl} / \mathrm{C}$ ratio and uniform element distributions in CNPs are explained by the violent fragmentation of DCM by high density $\mathrm{e}^{-}{ }_{\text {aq }}$ followed by the elimination of chlorine atom not only as $\mathrm{Cl}^{-}$but also $\mathrm{Cl}_{2}$.

\section{Materials and methods}

Dichloromethane (DCM, Nacalai Tesque, 99.5\%) and distilled water (Nacalai Tesque) was used without further purification. The saturated aqueous DCM solution $\left(0.15 \mathrm{~mol} \mathrm{dm}^{-3}\right)$ was prepared by separating the water phase one day after the mixing of DCM and water by shaking. The DCM/water biphasic solution was prepared by slowly adding $1 \mathrm{~mL}$ water to $1 \mathrm{~mL}$ of DCM in a quartz cuvette without disturbing the interface. Aqueous $\mathrm{HCl}$ (Nacalai Tesque) and $\mathrm{NaOH}$ 
(Nacalai Tesque) were used to prepare acidic $(\mathrm{pH} 2)$ and alkaline $(\mathrm{pH} \mathrm{10)}$ aqueous DCM solutions, respectively.

Femtosecond laser pulses $(0.8 \mu \mathrm{m}, 40 \mathrm{fs}, 0.4 \mathrm{~mJ}, 1 \mathrm{kHz})$ were focused on a quartz cuvette with a 1-cm optical path length by using a plano-convex quartz lens with a focal length of $50 \mathrm{~mm}$. The beam was focused on the center of the aqueous DCM solution $(1 \mathrm{~mL})$. In the case of the $\mathrm{DCM} /$ water biphasic solution, the beam was focused on the water phase $13 \mathrm{~mm}$ above the bottom of the cuvette (near the $\mathrm{DCM} /$ water interface).

The time evolution of the transmittance was measured by using a diode laser $(635 \mathrm{~nm})$ and a calibrated Si pin-photodiode. A pin-hole $(600 \mu \mathrm{m}$ in diameter) was placed in both the front and back of the cuvette. The output signal from a Si pin-photo diode was collected by using a data logger (Graphtec, GL820). To avoid any disturbance caused by bubbles, the transmittance measurement was carried out $30 \mathrm{~s}$ after the beam was blocked. Batch-type $\mathrm{pH}$ measurements were carried out by using a calibrated $\mathrm{pH}$ meter (Horiba Scientific, B-712, $12>\mathrm{pH}>2, \pm 0.1 \mathrm{pH}$ ).

After the 10-min laser irradiation, $5 \mu \mathrm{L}$ of aqueous DCM solution was dropped onto a copper grid covered with amorphous carbon film. The drop was allowed to dry in order to prepare specimens for transmission electron microscopic (TEM, JEOL, JEM-1010, $80 \mathrm{kV}$ ) observations.

In the case of the DCM/water biphasic solution, after the 10-min laser irradiation the solution was shaken to move the particles to the DCM phase. The DCM phase was then collected, centrifuged repeatedly $(13,500 \mathrm{rpm}, 90 \mathrm{~min})$, and dried. Particles were washed with water in order to remove $\mathrm{Na}$ and $\mathrm{Cl}$ atoms physically deposited on the surface of particles. The particles were sonicated in distilled water and centrifuged repeatedly. The particles were then separated from water followed by drying in a vacuum. The precipitates suspended in acetone were dropped onto a silicon substrate. The drop was allowed to dry in order to prepare samples for observation 
under a scanning electron microscope equipped with an energy-dispersive X-ray spectrometer (SEM-EDS, JEOL, JSM-6610LA, 15 kV) and a Raman spectrometer (JASCO, NRS-3100, 532 $\mathrm{nm})$.

\section{Results}

Figure 1 shows the aqueous DCM solution and the DCM/water biphasic solution after the 10min laser irradiation. It should first be mentioned that visible particles were not observed when DCM was exposed to laser pulses under our experimental conditions. Therefore, it is suggested that the excitation and/or ionization of water is essential since the excitation and/or ionization of DCM does not lead to particle formation. It is also noted that the DCM/water biphasic solution contained more black particles than the aqueous DCM solution. This trend was similar to that observed in our previous study: more carbon particles were produced in the organic/water biphasic solution than in the aqueous organic solution $[8,13]$. As has been proposed in the case of the organic/water biphasic solution $[8,13]$, the continuous supply of a carbon source (DCM in this study) through the DCM/water interface during the laser irradiation enhanced the production of particles. The black particles formed in the aqueous DCM solution moved to the DCM phase when DCM was added to the solution followed by shaking of the solution by hand. In the $\mathrm{DCM} /$ water biphasic solution, the black particles initially appeared in the water (upper) phase (Fig. 1b). However, these particles moved to the DCM (lower) layer when the DCM/water biphasic solution was shaken by hand. Therefore, it is reasonable to say that the black particles formed in both solutions were hydrophobic. 


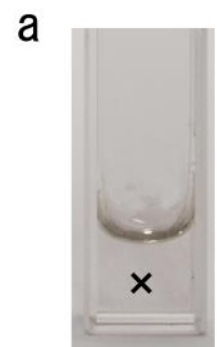

2.0

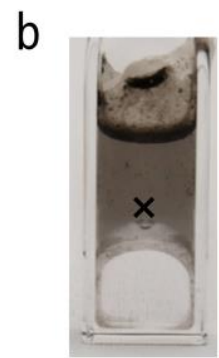

2.0

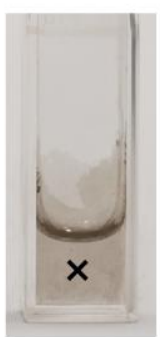

6.4

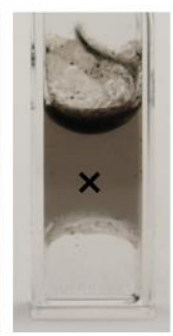

6.2

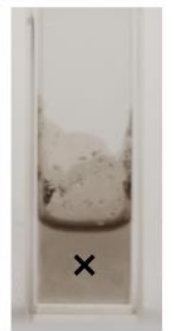

10.1

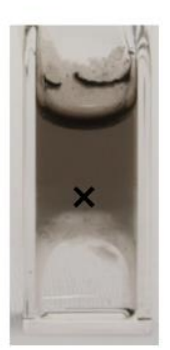

10.2

Figure 1. The cuvette after the 10-min laser irradiation. (a) Aqueous DCM solution, (b) $\mathrm{DCM} /$ water biphasic solution. The values below the images indicate the $\mathrm{pH}$ before irradiation. Laser focusing position is indicated by $\times$.

Laser irradiation was carried out for samples with different initial $\mathrm{pH}$ values. It was obvious that less amount of black particles were formed in the acidic than in the alkaline aqueous DCM solution (Fig. 1a). In the DCM/water biphasic solution, on the other hand, no initial pH effect was visible due to the high optical density of particles (Fig. 1b). The estimation of particle amount by weight is difficult because the 10-min laser irradiation gives less than $0.1 \mathrm{mg}$ of particles although particles seem to be formed in high density. Therefore, we measured transmittance in order to trace the particle formation processes. Figure 2 shows the time evolution of the transmittance measured at $635 \mathrm{~nm}$. It should be noted that the condensation of black particles on the air-liquid interface (Fig. 1) prevents us from evaluating the actual amounts 
of black particles. The condensation behavior was observed after the 5-min (aqueous DCM solution) and the 3-min (DMC/water biphasic solution) laser irradiation. The transmittances of aqueous DCM solution after the 5-min laser irradiation were 69\% (initial pH was 2.1 ), $40 \%$ (initial $\mathrm{pH}$ was 6.2 ), and $38 \%$ (initial $\mathrm{pH}$ was 9.9). These results are consistent with what was observed by the eye (Fig. 1a). The transmittance seems to become constant by prolonged irradiation judging from the decay behavior in the cases of aqueous DCM solution.
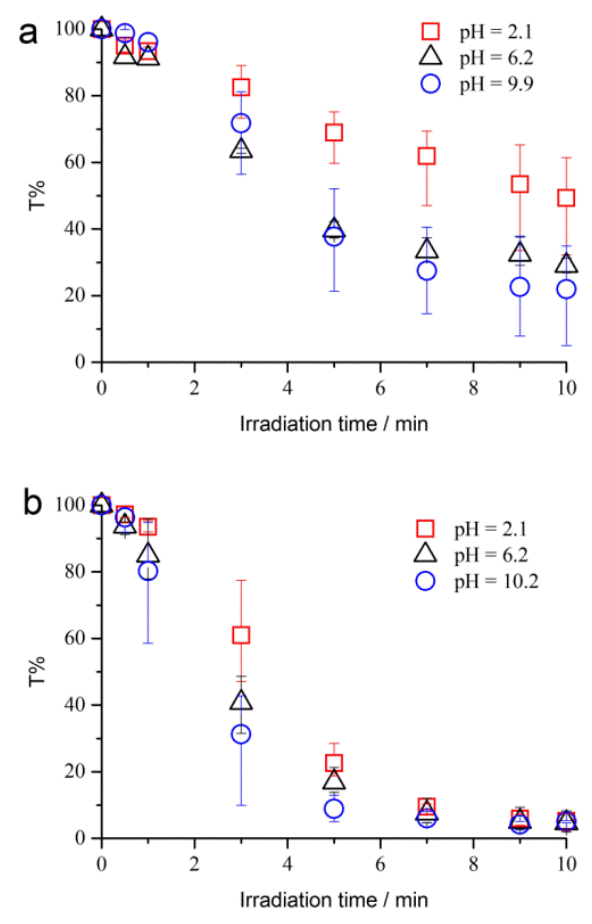

Figure 2. The time evolutions of the transmittance of (a) aqueous DCM solution and (b) the water phase of the $\mathrm{DCM} /$ water biphasic solution. The initial $\mathrm{pH}$ was adjusted to ca. 2, 6, or 10 . The vertical bars show the maximum and minimum values obtained in the three measurements.

The decrease in the transmittance of the water phase was faster in the $\mathrm{DCM} /$ water biphasic solution than in the aqueous DCM solution. The transmittances of the water phase of the $\mathrm{DMC} /$ water biphasic solution after the 3-min laser irradiation, in which the condensation was not 
visible, were $61 \%$ (initial $\mathrm{pH}$ was 2.1 ), $41 \%$ (initial $\mathrm{pH}$ was 6.2 ), and $31 \%$ (initial $\mathrm{pH}$ was 10.2 ). The transmittance of the acidic solution was almost twice that of the alkaline solution. However, the transmittance became almost zero, independent on the initial $\mathrm{pH}$ value, after the 10 -min laser irradiation, as confirmed by the eye (Fig. 1b). Although the quantitative analysis on reaction rates is hardly difficult by means of transmittance measurement, it takes longer time to reach $50 \%$ transmittance under acidic condition compared with that under alkaline condition. The times required to reach $50 \%$ transmittance of the aqueous DCM solution were $10(\mathrm{pH} 2.1), 4.1$ (pH 6.2), and $4.3 \mathrm{~min}(\mathrm{pH} 9.9$ ). Those of the DCM/water biphasic solution were 3.3 (pH 2.1), 2.4 (pH 6.2), and $2.1 \mathrm{~min}(\mathrm{pH} \mathrm{10.2).} \mathrm{Although} \mathrm{there} \mathrm{are} \mathrm{some} \mathrm{ambiguities} \mathrm{in} \mathrm{the} \mathrm{transmittance}$ measurements, it is concluded that the rate and yield of black particle formation is suppressed under acidic conditions.
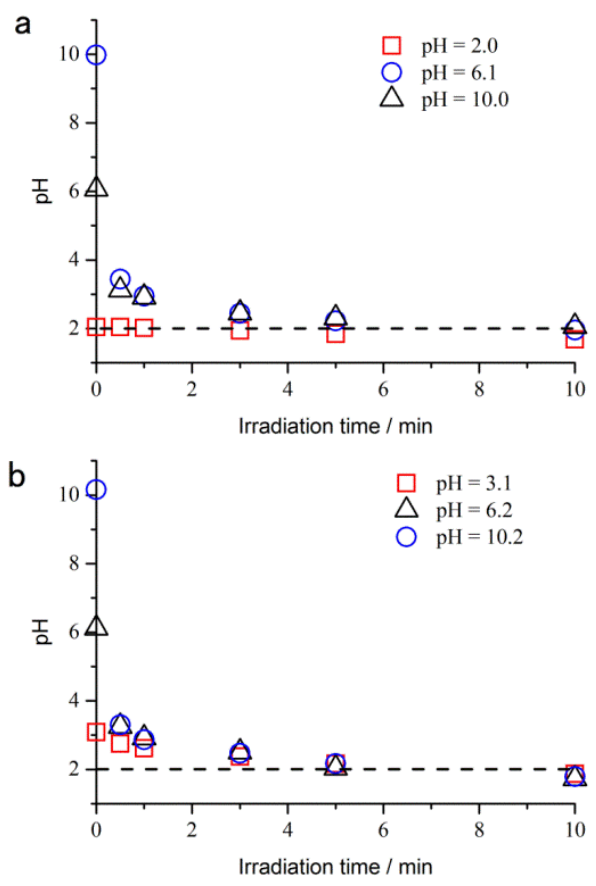

Figure 3. The time evolution of $\mathrm{pH}$. (a) Aqueous DCM solution, (b) DCM/water biphasic solution. Broken lines indicate the lower limit of $\mathrm{pH}$ measurement. 
The time evolution of $\mathrm{pH}$ upon laser irradiation is shown in Fig. 3. The $\mathrm{pH}$ dropped to around 3 by the 30 -s irradiation. This sudden drop is independent on the initial $\mathrm{pH}$ in both the aqueous DCM solution and the DCM/water biphasic solution. In both cases, the $\mathrm{pH}$ became 2, which was the lower limit of our $\mathrm{pH}$ measurement, by the 5-min irradiation. The decrease in $\mathrm{pH}$ indicates the formation of $\mathrm{Cl}^{-}$as the counter ion of $\mathrm{H}^{+}$. The addition of silver nitrate aqueous solution to the DCM/water biphasic solution as well as to the aqueous DCM solution gave white precipitates after the 10-min laser irradiation. Furthermore, the coloration of the reaction solution occurred by adding a chlorine indicator such as 4,4'-diamino-3,3'-dimethylbiphenyl (or N,N-diethyl-pphenylenediamine) aqueous solution. These results indicate that the reaction solution after laser irradiation contains $\mathrm{Cl}^{-}$as well as $\mathrm{Cl}_{2}$ and/or $\mathrm{HClO}$.

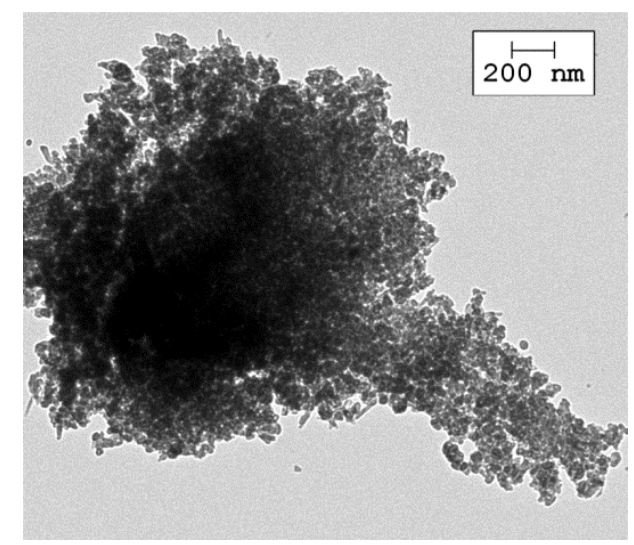

Figure 4. TEM image of the black particles collected from the aqueous DCM solution (initial $\mathrm{pH}$ $=6.0)$.

Figure 4 shows a typical transmission electron microscope (TEM) image of black particles produced by the 10-min irradiation to the aqueous DCM solution. Most of the particles were agglomerated, but the individual particles were less than $50 \mathrm{~nm}$ in diameter. The size and 
morphology seemed to be independent of the initial $\mathrm{pH}$ and the reaction solution used (aqueous DCM solution or DCM/water biphasic solution) although the size distribution was not obtained due to large agglomeration. Figure 5 shows the Raman spectra of the black particles. Two significant bands in the spectra were assigned as a D band (ca. $1340 \mathrm{~cm}^{-1}$ ) and a $\mathrm{G}$ band (ca. $1580 \mathrm{~cm}^{-1}$ ), respectively. It is clear that the particles show typical peculiarities of disordered structure of CNPs. The peak position and the ratio between the D band and G band intensities seemed to be independent of the initial $\mathrm{pH}$.

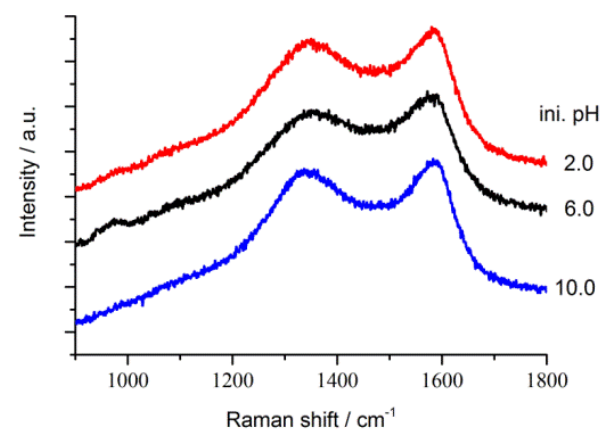

Figure 5. Raman spectra of black particles.

Table 1 shows the atomic composition as well as the $\mathrm{Cl} / \mathrm{C}$ and $\mathrm{O} / \mathrm{C}$ atomic ratios of CNPs obtained by a scanning electron microscope equipped with an energy-dispersive X-ray spectrometer (SEM-EDS). Due to the deep penetration of high-energy electrons, the SEM-EDS measurements can be used to investigate the atomic composition of agglomerated whole particles. In addition, the irradiation area of electrons $\left(100 \times 100 \mu \mathrm{m}^{2}\right)$ was large enough to allow us to obtain the averaged atomic composition of the CNPs. It was found that both the $\mathrm{Cl} / \mathrm{C}$ and $\mathrm{O} / \mathrm{C}$ atomic ratios were independent of the initial $\mathrm{pH}$. We emphasize that the $\mathrm{Cl} / \mathrm{C}$ atomic ratio is reduced from $2\left(\mathrm{CH}_{2} \mathrm{Cl}_{2}\right)$ to $0.05-0.06$ (products). The obtained atomic compositions correspond 
to a particle with an approximate $\mathrm{C}_{35} \mathrm{O}_{3} \mathrm{Cl}_{2}$ stoichiometry. Furthermore, the elemental mapping analysis using SEM-EDS showed the uniform distribution of each element in the particles (Fig. $6)$.

Table 1. Atomic compositions (atom $\%$ ) and their relative ratios of carbon nanoparticles

\begin{tabular}{cccccc}
\hline $\mathrm{pH}$ & $\mathrm{C}^{\mathrm{a}}$ & $\mathrm{O}^{\mathrm{a}}$ & $\mathrm{Cl}^{\mathrm{a}}$ & $\mathrm{O} / \mathrm{C}$ & $\mathrm{Cl} / \mathrm{C}$ \\
\hline 2 & $88.1 \pm 0.7$ & $6.7 \pm 0.6$ & $5.0 \pm 0.3$ & 0.076 & 0.057 \\
6 & $88.3 \pm 1.0$ & $7.2 \pm 0.9$ & $4.4 \pm 0.3$ & 0.081 & 0.050 \\
10 & $88.3 \pm 1.0$ & $6.8 \pm 1.4$ & $4.8 \pm 1.0$ & 0.077 & 0.054
\end{tabular}

${ }^{a}$ Average of six $(\mathrm{pH}=2,6)$ and four $(\mathrm{pH}=10)$ measurements.
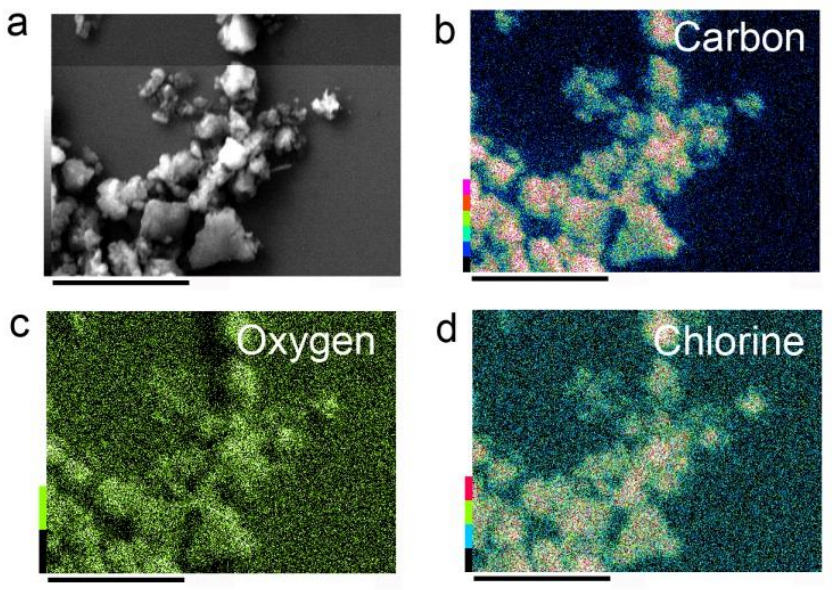

Figure 6. (a) SEM-image and (b-d) EDS mappings of carbon particles collected from the aqueous DCM solution (initial $\mathrm{pH}=6.0$ ). Scale bar, $20 \mu \mathrm{m}$. 


\section{Discussion}

We demonstrated the transformation of DCM dissolved in water to low-chlorine CNPs by femtosecond laser irradiation. In order to clarify the plausibility of the femtosecond laser pulses in the production of low-chlorine CNPs from DCM, we need to consider 1) what was the reactive species and primary reaction, 2) why did the suppression of CNP production occur under acidic conditions, 3) why did $\mathrm{Cl}_{2}$ (or $\mathrm{HClO}$ ) exist in the reaction solution, 4) why did CNPs contain small amounts of chlorine, 5) why was each element uniformly distributed in particles, and 6) how we could improve the treatment efficiency?

By the exposure of water to femtosecond laser pulses, reactive species such as $\mathrm{e}^{-}$aq, $\bullet \mathrm{OH}$, and $\mathrm{H} \cdot$ are generated. They react with DCM as described in Scheme 1. The reaction rate of DCM with $\mathrm{e}_{\text {aq }}^{-}(1), \cdot \mathrm{OH}(2)$, and $\mathrm{H} \bullet(3)$ are $6.3 \times 10^{9}[14], 6.6 \times 10^{7}[15]$, and $4.0 \times 10^{6} \mathrm{dm}^{3} \mathrm{~mol}^{-1} \mathrm{~s}^{-1}[14]$, respectively. Femtosecond laser irradiation produces significantly high density of electrons, which is estimated to be from $10^{18}\left(1.7 \times 10^{-3} \mathrm{~mol} \mathrm{dm}^{-3}[16]\right)$ to $7.3 \times 10^{19} \mathrm{~cm}^{-3}\left(0.12 \mathrm{~mol} \mathrm{dm}^{-3}\right.$ [17]). The highest value is presumably incorrect because the annihilation before hydration is not considered [17], however, even the lowest value is far exceed the concentration of $\mathrm{e}^{-}$aq (on the order of $\mu \mathrm{mol} \mathrm{dm}{ }^{-3}[18],<4 \mu \mathrm{mol} \mathrm{dm}{ }^{-3}$ at $\left.7 \mathrm{~Gy}[5]\right)$ that obtained by radiolysis. Based on the rate constants of DCM mentioned above and the expected electron density, the reaction between DCM and $\mathrm{e}^{-}$aq would be the most probable primary reaction. Unfortunately, the chemistry beyond the primary reactions between DCM and $\mathrm{e}^{-}$aq is uncertain due to its complexity. It is noted that $\mathrm{e}^{-}$aq also attacks hexafluorobenzene dissolved in water to form CNPs [19]. 


$$
\begin{aligned}
& \mathrm{DCM}+\mathrm{e}^{-} \mathrm{aq} \longrightarrow \cdot \mathrm{CH}_{2} \mathrm{Cl}+\mathrm{Cl}^{-} \\
& \mathrm{DCM}+\cdot \mathrm{OH} \longrightarrow \cdot \mathrm{CHCl}_{2}+\mathrm{H}_{2} \mathrm{O} \\
& \mathrm{DCM}+\cdot \mathrm{H} \longrightarrow \cdot \mathrm{CHCl}_{2}+\mathrm{H}_{2} \cdot \mathrm{CH}_{2} \mathrm{Cl}+\mathrm{HCl} \\
& \mathrm{e}^{-}{ }_{\text {aq }}+\mathrm{e}^{-}{ }_{\text {aq }} \underset{\mathrm{H}_{2} \mathrm{O}}{\longrightarrow} \mathrm{H}_{2}+2 \mathrm{OH}^{-} \\
& \mathrm{e}^{-}{ }_{\mathrm{aq}}+\cdot \mathrm{H} \quad \underset{\mathrm{H}_{2} \mathrm{O}}{\longrightarrow} \mathrm{H}_{2}+\mathrm{OH}^{-} \\
& \mathrm{e}^{-}{ }_{\mathrm{aq}}+\cdot \mathrm{OH} \longrightarrow \mathrm{OH}^{-} \\
& \mathrm{e}^{-}{ }_{\mathrm{aq}}+\mathrm{O}_{2} \longrightarrow \cdot \mathrm{O}_{2}^{-} \\
& \mathrm{e}^{-} \mathrm{aq}+\mathrm{H}^{+} \longrightarrow \cdot \mathrm{H} \\
& \mathrm{Cl}^{-} \longrightarrow \cdot \mathrm{Cl}+\mathrm{e}^{-} \text {aq } \\
& \cdot \mathrm{Cl}+\cdot \mathrm{Cl} \longrightarrow \mathrm{Cl}_{2} \\
& \cdot \mathrm{Cl}+\mathrm{Cl}^{-} \longrightarrow \cdot \mathrm{Cl}_{2}^{-}
\end{aligned}
$$

Scheme 1. Possible Reactions in Aqueous DCM.

In order to clarify the $\mathrm{pH}$ effect on the production of CNPs, we consider the quenching reactions of $\mathrm{e}^{-}$aq competitive with the primary reaction between DCM and $\mathrm{e}^{-}$aq. The rate of DCM disappearance by $\mathrm{e}_{\text {aq }}^{-}$is $9.5 \times 10^{8} \mathrm{~s}^{-1}(1.1 \mathrm{~ns})$ based on the saturated concentration of DCM in water $\left(0.15 \mathrm{~mol} \mathrm{dm}^{-3}\right)$. The quenching reaction rate constants of $\mathrm{e}^{-}$aq with $\mathrm{e}^{-}$aq $(4), \mathrm{H} \bullet(5), \cdot \mathrm{OH}$ (6), $\mathrm{O}_{2}(7)$, and $\mathrm{H}^{+}(8)$ are $0.55 \times 10^{10}, 2.5 \times 10^{10}, 3.0 \times 10^{10}, 1.9 \times 10^{10}$, and $2.3 \times 10^{10} \mathrm{dm}^{3} \mathrm{~mol}^{-1} \mathrm{~s}^{-1}$, respectively [20]. The reactions (4)-(6) would influence the $\mathrm{pH}$ of the solution because $\mathrm{OH}^{-}$is formed in these reactions. However, the $\mathrm{pH}$ of the solution fell from 6 or 10 to 3 with the 30 -s laser irradiation, as shown in Fig. 3. The degree of $\mathrm{pH}$ drop indicates that the solution is not neutralized efficiently by $\mathrm{OH}^{-}$. Therefore, the annihilation (4) and scavenging process of $\mathrm{e}^{-}$aq by $\mathrm{H} \cdot(5)$ and/or $\bullet \mathrm{OH}(6)$ are expected to be minor processes. The concentration of $\mathrm{O}_{2}$ in water is $2.7 \times 10^{-4} \mathrm{~mol} \mathrm{dm}{ }^{-3}$, thus the rate of $\mathrm{e}^{-}$aq disappearance and the formation of $\mathrm{O}_{2}^{-} \cdot$ under aerated conditions is $5.1 \times 10^{6} \mathrm{~s}^{-1}(0.19 \mu \mathrm{s})$. Though $\mathrm{O}_{2}$ is evolved from water by a prolonged 
femtosecond laser irradiation [21], the quenching of $\mathrm{e}^{-}$aq by oxygen (7) would not be important. The rate of $\mathrm{e}^{-}$aq disappearance by $\mathrm{H}^{+}$is calculated to be $2.3 \times 10^{8} \mathrm{~s}^{-1}(4.3 \mathrm{~ns})$ when $\mathrm{pH}$ is 2 . Thus, this reaction (8) is competitive with the disappearance of $\mathrm{e}^{-}$aq by DCM (1.1 ns). In addition, the resultant $\mathrm{H}^{\bullet}$ is inactive toward DCM. Based on the kinetics of $\mathrm{e}^{-}$aq, it is concluded that the suppression of CNP formation under acidic conditions is attributable to the loss of $\mathrm{e}^{-}$aq by $\mathrm{H}^{+}$.

Although neither $\mathrm{Cl}_{2}$ nor $\mathrm{HClO}$ was detected in high-dose radiolysis [5], we detected $\mathrm{Cl}_{2}$ (or $\mathrm{HClO}$ ) as well as $\mathrm{Cl}^{-}$in the reaction solution after the 10-min laser irradiation. The detection of $\mathrm{Cl}_{2}$ (or $\mathrm{HClO}$ ) in this study indicates that it is necessary to consider the transformation of $\mathrm{Cl}^{-}$to $\mathrm{Cl}_{2}$ (or $\mathrm{HClO}$ ). However, $\mathrm{Cl}^{-}$does not transform to $\mathrm{Cl}_{2}$ without strong oxidizing reagents. The possible process by which to change the valence of $\mathrm{Cl}^{-}$may be the multiphoton ionization of $\mathrm{Cl}^{-}$ to $\mathrm{Cl} \bullet(9)$ by femtosecond laser pulses. Once $\mathrm{Cl} \bullet$ is formed, $\mathrm{Cl}_{2}$ may presumably be formed by the recombination of two $\mathrm{Cl} \bullet(10)$. However, $\mathrm{Cl} \bullet$ probably reacts with $\mathrm{Cl}^{-}$, forming $\cdot \mathrm{Cl}_{2}^{-}(11$, $8.5 \times 10^{9} \mathrm{dm}^{3} \mathrm{~mol}^{-1} \mathrm{~s}^{-1}$ [22]), because the self-recombination of $\mathrm{Cl} \bullet$ would be a minor process [22]. Once $\bullet \mathrm{Cl}_{2}{ }^{-}$is formed, $\mathrm{Cl}_{2}$ is produced from $\mathrm{Cl} \cdot$ and $\bullet \mathrm{Cl}_{2}{ }^{-}$, and also from two $\bullet \mathrm{Cl}_{2}{ }^{-}$[22]. More probably, multiphoton ionization of $\cdot \mathrm{Cl}_{2}^{-}$forms $\mathrm{Cl}_{2}$ since $\bullet \mathrm{Cl}_{2}{ }^{-}$has absorption maximum at 340 $\mathrm{nm}$, which is accessible by three $0.8-\mu \mathrm{m}$ photons. The $\mathrm{pK}\left(\left[\mathrm{Cl}_{2}\right] /[\mathrm{HClO}]=1\right)$ varies from 1.25 to 2.11, depending on the concentration of chlorine [23]. In addition, the solubility of $\mathrm{Cl}_{2}$ in water is high $\left(\approx 0.08 \mathrm{~mol} \mathrm{dm}{ }^{-3}\right.$ at $\left.\mathrm{pH}=2\right)$ even in the presence of $\mathrm{HCl}[24]$. Thus $\mathrm{Cl}^{-}$as well as $\mathrm{Cl}_{2}$ was the dominant form of chlorine after the 5-min laser irradiation, as the $\mathrm{pH}$ of the reaction solution became 2. Although we cannot evaluate the concentration of $\mathrm{Cl}_{2}$ by absorption spectroscopy due to the dense formation of CNPs, it is suggested that the elimination of chlorine atom not only as $\mathrm{Cl}^{-}$but also $\mathrm{Cl}_{2}$ would presumably be one of the important factor in low-chlorine products 
formation. The efficient elimination of chlorine atoms baring carbon skeleton of DCM helps carbon network constructions and finally leads to CNP formation.

The partial fragmentation of DCM would leave chlorine atoms in the fragments. Thus, the recombination of these fragments results in dimeric compounds such as $\mathrm{C}_{2} \mathrm{H}_{4} \mathrm{Cl}_{2}$ [6] as well as a chlorine-incorporated polymer found in radiolysis [5]. If the CNPs are formed by femtosecond laser irradiation via the partial fragmentation processes, the resultant CNPs should contain large amounts of chlorine. The elemental analysis, however, showed that only a small portion of chlorine was incorporated into the resultant CNPs $\left(\mathrm{Cl} / \mathrm{C}=0.06, \mathrm{C}_{35} \mathrm{O}_{3} \mathrm{Cl}_{2}\right)$. In addition, each element was uniformly distributed in CNP agglomerates. Therefore, it is suggested that the lowchlorine CNP formation is explained in terms of the violent fragmentation of DCM and/or its fragments by femtosecond laser pulses.

The precipitation of DCM as low-chlorine CNPs in water is demonstrated by femtosecond laser irradiation. This evidence reveals the potential ability of femtosecond laser processing of organic wastes dissolved in water. However, our proposed method has many difficulties for the engineering applications (productivity, high investment cost, and scalability). The low production yield is originated in the very small reaction volume (in a few tens of micrometers in diameter [12]) although the electron density achieved in this study reaches $10^{18} \mathrm{~cm}^{-3}\left(1.7 \times 10^{-3}\right.$ mol $\mathrm{dm}^{-3}$.) In addition, laser pulses cannot penetrate due to the high optical density when the black CNPs are formed. These disadvantages could be overcome by producing several hundreds of focusing spots using a fly's-eye lens array as well as by using a flow reactor $[25,26]$. More cost effective and high productivity approach may be the replacement of femtosecond laser to the combination of other plasma sources and VUV light if high density electron generation as well as successive photoreactions are possible. 


\section{Conclusions}

The precipitation of DCM as low-chlorine CNPs in water is demonstrated for the first time by femtosecond laser irradiation. The production of the agglomerates of CNPs was suppressed under low $\mathrm{pH}$ conditions owing to the quenching of the primary reduction reaction between DCM and $\mathrm{e}^{-}$aq by protons. The complete destruction of the DCM skeleton excluding chlorine atom from the CNPs construction process enables us to produce low-chlorine CNPs. We propose that the generation of high density of electron as well as successive multiphoton reactions only achievable by femtosecond laser pulses is a useful and alternative way to remove CHCs from water.

\section{Acknowledgements}

This research was supported financially in part by JSPS KAKENHI Grant Numbers JP26620014, JP24227002, JP26107002. We thank Mr. Yuhei Tahara and Prof. Makoto Miyata for their help with TEM experiments.

\section{References}

[1] M. Shestakova, M. Sillanpää, Removal of dichloromethane from ground and wastewater: A review, Chemosphere, 93 (2013) 1258-1267. 
[2] R. W. Gillham, S. F. Ohannesin, Enhanced degradation of halogenated alophatics by zero-valent iron, Groundwater, 32 (1994) 958-967.

[3] A. Bhatnagar, H. M. Cheung, Sonochemical destruction of chlorinated C1 and C2 volatile organic compounds in dilute aqueous solution, Environ. Sci. Technol., 28 (1994) $1481-1486$.

[4] H-R. Park, Y-T. Jeong, M-S. Kim, H-G. Woo, H-S. Ham, Photochemical reaction of dichloromethane in aqueous solution, Bull. Korean Chem. Soc., 18 (1997) 287-291.

[5] P. Rajesh, J. A. LaVerne, S. M. Pimblott, High dose radiolysis of aqueous solutions of chloromethanes: Importance in the storage of radioactive organic wastes, J. Nucl. Mater., 361 (2007) 10-17.

[6] V. S. Kosobutskii, A. I. Vrublevskii, Transformations of dichloromethane radicals in alkaline water solutions, Russ. J. Org. Chem., 38 (2002) 475-479.

[7] T. Yatsuhashi, N. Uchida, K. Nishikawa, Novel method of producing carbon nanoparticles on benzene/water interface with femtosecond laser plasma filament, Chem. Lett., $41(2012) 722-724$.

[8] T. Hamaguchi, T. Okamoto, K. Mitamura, K. Matsukawa, T. Yatsuhashi, Synthesis of hydrophilic and hydrophobic carbon nanoparticles from benzene/water bilayer solution with femtosecond laser generated plasma filaments in water, Bull. Chem. Soc. Jpn., 88 (2015) $251-261$.

[9] D. Nishida, M. Kusaba, T. Yatsuhashi, N. Nakashima, Reduction of $\mathrm{Eu}^{3+}$ to $\mathrm{Eu}^{2+}$ by an intense femtosecond laser pulse in solution, Chem. Phys. Lett., 465 (2008) 238-240. 
[10] D. Nishida, E. Yamade, M. Kusaba, T. Yatsuhashi, N. Nakashima, Reduction of $\mathrm{Sm}^{3+}$ to $\mathrm{Sm}^{2+}$ by an intense femtosecond laser pulse in solution, J. Phys. Chem. A, 114 (2010) $5648-5654$.

[11] N. Nakashima, K. Yamanaka, T. Yatsuhashi, Chin. J. Phys., 52 (2014) 504-518.

[12] N. Nakashima, K. Yamanaka, M. Saeki, H. Ohba, S. Taniguchi, T. Yatsuhashi, Metal ion reductions by femtosecond laser pulses with micro-Joule energy and their efficiencies, J. Photochem. Photobiol. A, 319 (2016) 70-77.

[13) E. Miyasaka, T. Hamaguchi, T. Yatsuhashi, Graphitization of chlorohydrocarbons in laser-induced plasma filaments, IEEJ Transactions on Electronics, Information and Systems, 135 (2015) 1075-1079.

[14] P. Calza, E. Pelizzetti, Reactivity of chloromethanes with photogenerated hydrated electrons, J. Photochem. Photobiol. A, 162 (2004) 609-613.

[15] E. Villenave, V. L. Orkin, R. E. Huie, M. J. Kurylo, Rate constant for the reaction of OH radicals with dichloromethane, J. Phys. Chem. A, 101 (1997) 8513-8517.

[16] W. Liu, O. Kosareva, I. S. Golubtsov, A. Iwasaki, A. Becker, V. P. Kandidov, S. L. Chin, Femtosecond laser pulse filamentation versus optical breakdown in $\mathrm{H}_{2} \mathrm{O}$, Appl. Phys. B, 76 (2003) 215-229.

[17] F. V. Potemkin, E. I. Mareev, Dynamics of multiple bubbles, excited by a femtosecond filament in water, Laser Phys. Lett., 12 (2015) 015405.

[18] S. Le Caër, Water radiolysis: influence of oxide surfaces on $\mathrm{H}_{2}$ production under ionizing radiation, Water, 3 (2011) 235-253. 
[19] T. Okamoto, K. Mitamura, T. Hamaguchi, K. Matsukawa, T. Yatsuhashi, ChemPhysChem, https://doi.org/ 10.1002/cphc.201600602.

[20] G. V. Buxton, C. L. Greenstock, W. P. Helman, A. B. Ross, Critical-review of rate constants for reactions of hydrated electrons, hydrogen-atoms and hydroxyl radicals $\left(\bullet \mathrm{OH} / \bullet \mathrm{O}^{-}\right)$ in aqueous-solution, J. Phys. Chem. Ref. Data, 17 (1988) 513-886.

[21] S. L. Chin, S. Lagace, Generation of $\mathrm{H}_{2}, \mathrm{O}_{2}$, and $\mathrm{H}_{2} \mathrm{O}_{2}$ from water by the use of intense femtosecond laser pulses and the possibility of laser sterilization, Appl. Opt., 35 (1996) 907-911.

[22] G. V. Buxton, M. Bydder, G. A. Salmon, Reactivity of chlorine atoms in aqueous solution - Part 1 The equilibrium Cl• $+\mathrm{Cl}^{-} \rightarrow \leftarrow \mathrm{Cl}_{2}{ }^{\bullet}$, J. Chem. Soc. Faraday Trans., 94 (1998) $653-657$.

[23] D. P. Cherney, S. E. Duirk, J. C. Tarr, T. W. Collette, Monitoring the speciation of aqueous free chlorine from $\mathrm{pH} 1$ to 12 with Raman spectroscopy to determine the identity of the potent low-pH oxidant, Appl. Spectrosc., 60 (2006) 764-772.

[24] M. Alkan, M. Oktay, M. M. Kocakerim, M. Copur, Solubility of chlorine in aqueous hydrochloric acid solutions, J. Hazard Mater., 119 (2005) 13-18.

[25] R. Streubel, S. Barcikowski, B. Gökce, Continuous multigram nanoparticle synthesis by high-power, high-repetition-rate ultrafast laser ablation in liquids, Opt. Lett., 41 (2016) 120-130.

[26] Mttaqin, T. Nakamura, S. Sato, Synthesis of gold nanoparticle colloids by highly intense laser irradiation of aqueous solution by flow system, Appl. Phys. A, 120 (2015) 881-888. 